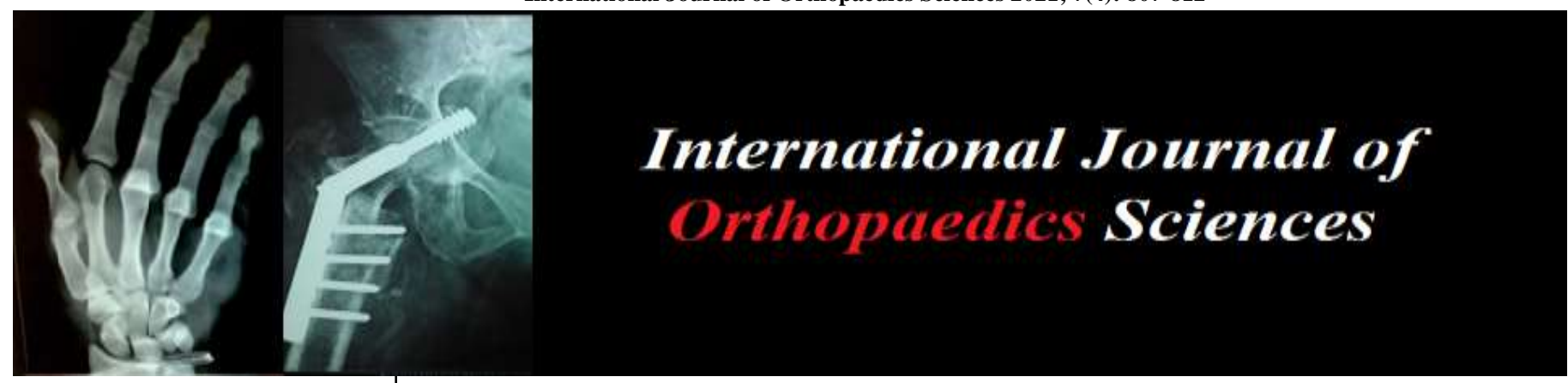

E-ISSN: 2395-1958

P-ISSN: 2706-6630

IJOS 2021; 7(4): 807-812

(C) 2021 IJOS

www.orthopaper.com

Received: 15-07-2021

Accepted: 03-09-2021

Ayush Sharma

Department of Orthopaedic

Surgery, Indira Gandhi Medical

College, Shimla, Himachal

Pradesh, India

Mukand Lal

Department of Orthopaedic

Surgery, Indira Gandhi Medical

College, Shimla, Himachal

Pradesh, India

Sandeep Kashyap

Department of Orthopaedic

Surgery, Indira Gandhi Medical

College, Shimla, Himachal

Pradesh, India

\section{Anupam Jhobta}

Department of Radio-diagnosis, Indira Gandhi Medical College,

Shimla, Himachal Pradesh,

India
Corresponding Author:

Ayush Sharma

Department of Orthopaedic

Surgery, Indira Gandhi Medical

College, Shimla, Himachal

Pradesh, India

\section{Radiological comparison of acetabular anteversion on anteroposterior roentgenograms and computed tomograms \& functional outcomes in total hip arthroplasty}

\author{
Ayush Sharma, Mukand Lal, Sandeep Kashyap and Anupam Jhobta
}

DOI: $\underline{\text { https://doi.org/10.22271/ortho.2021.v7.i41.2970 }}$

\section{Abstract}

Introduction: Orientation and Alignment of prosthetic components are vitally important for the stability of total hip arthroplasty. Poor acetabular positioning is one of the many issues implicated with persistent pain due to impingement, dislocation, edge loading and liner fracture, which may be lead to patient dissatisfaction after total hip arthroplasty.

Material and methods: Post-operative radiological analysis of the version of acetabulum through X-ray images and CT images was performed. Pre \& post-operative scoring according to Modified Harris Hip Score (HHS), Western Ontario and McMaster Universities Osteoarthritis Index (WOMAC) and Oxford Hip Score (OHS) was documented.

Results: A total of 55 patients were included in the study. The mean anteversion angle calculated on anteroposterior (AP) radiographs by Lewinnek's method was 23.480 (Range $11-390$ ), compared to CT Scans measured was 28.640 (Range $11.10-50.100$ ).

Conclusion: Majority $69.09 \%$ of patients had excellent functional outcomes in a range of $11.1-360$ of anteversion compared to Lewinnek's safe zone. It suggests that there is flexibility in positioning the acetabular component than previously believed. If one has to err, it should be towards more anteversion. Infact to avoid dislocation, more anteversion is required to guard against unwarranted activities on part of the patient.

Keywords: Hip arthroplasty, lewinnek method, anteversion, biomechanics

\section{Introduction}

Total Hip Arthroplasty (THA) is one of the most common procedures performed in Orthopaedic Surgery and is considered to be one of the best medical innovations of our generation [1]. Arthroplasty depends upon ideal placement of both acetabular and femoral components. Accurate biomechanical reconstruction of the joint is essential to achieve function and longevity with acetabular positioning being a key factor, the consequences of malposition include instability, increased wear, impaired muscle function, reduced range of motion (ROM), impingement, bearing-related noise generation, poor functional outcomes, 1 limb length discrepancy, and loosening and cup failure ${ }^{[2,3,4]}$. Dislocation is one of the most frequent complications after THA with an incidence of $0.6 \%$ to $11 \%$ in the early postoperative period; between $13 \%$ and $30 \%$ of dislocations reportedly are caused by implant malpositioning ${ }^{[5]}$.It is found that even in normal subjects there is a great variation in acetabular morphology. However, the range of normal acetabular \& femoral variation may differ between races ${ }^{[6]}$. At age 13 to 14, the mostly ossified bones of the ilium, ischium, and pubis unite at the acetabulum, forming $\mathrm{Y}$-shaped triradiate cartilage that proceeds to fusion by age 15 to 16 . The other secondary centers unite and fuse between the ages of 20 and 22 . The acetabular surface is orientated approximately 45 degrees caudally and 15 degrees anteriorly. The average anteversion of the native acetabulum measures 16 to 21 degrees with an average inclination of 48 degrees. Men tend to have less anteversion than females. Angular position includes the anteversion and inclination (abduction angle) of the cup. The most commonly quoted study is by Lewinnek et al. 
He found an increased dislocation rate in cups placed outside anteversion angles of $5^{\circ}-25^{\circ}$ and $30^{\circ}-50^{\circ}$ of inclination. Inclination and anteversion can thus be operative, radiologic, or anatomic ${ }^{[7]}$. Operative and radiographic angles are the most relevant surgically in the lateral position of the patient. During surgery in the lateral position, operative anteversion is assessed by looking down, to project the insertion angle onto the sagittal plane, and measuring against the longitudinal axis. The most commonly used method for radiographic analysis is the one proposed by Woo and Morrey ${ }^{[8]}$ namely the "angle formed by a line drawn tangential to the face of the acetabulum and a line perpendicular to the horizontal plane, as seen on a lateral view of the pelvis". In comparison, McCollum and Gray [9] suggested a position of $40^{\circ} \pm 10^{\circ}$ abduction and $30^{\circ} \pm 10^{\circ}$ flexion to prevent impingement and dislocation. Harris recommends a position of $30^{\circ}$ abduction and $20^{\circ}$ anteversion; however, the Harris angles are referenced using a mechanical guide and the trunk of the patient ${ }^{[10]}$. During the implantation process, surgeons use different techniques to judge the positioning of the acetabular component. The purpose of the present study was aimed at the morphometric evaluation of the acetabular version radiologically using well-defined parameters of acetabular positioning in primary total hip replacements on patient satisfaction and functional outcomes at a tertiary center which will further help in planning, execution, and evaluation of total hip arthroplasty (THA). However, most of the studies have been done on western populations with limited followup period \& to the best of our knowledge, only limited studies have been done in the Indian populations. The present study was undertaken to study the effects of acetabular component position well as acetabular position relative to bony anatomy on patient-reported functional outcomes and further to compare results whether accurately positioned acetabular component in Lewinnek's safe zone using freehand technique as wide variability has been reported in cup orientation, also how accurately operating surgeon can achieve desired anteversion using factors such as visual cues and side of the operating table. To determine whether acetabular component positioned assessed by cross table radiographs and anteroposterior radiographs and computed tomography could provide measure of acetabular version and compare their reliability and accuracy of methods.

\section{Material and methods}

This present study was conducted in tertiary care institution between the periods of 2017 to 2019, a total of 55 patients were included in the study. In this study, males were $58.2 \%$ compared to $41.8 \%$ females. The mean age of study population was 49.65 years with range from $17-75$ years. The most common indication for surgery in our study was idiopathic osteoarthritis in $43.64 \%$ patients followed by postsurgical osteonecrosis of femoral head in $21.82 \%$. The mean follow-up was 19.83 months.

\section{Inclusion criteria}

- All age group patients, who need THA for painful disabling hip were included in the study.

\section{Exclusion criteria:}

- Revision surgery

- Patients with bony pathology of pelvis and femur

- Patients with contralateral hip pathology as evident clinically with gait abnormality and pain or restriction of movements.
- Patients with current or previous metabolic bone disease.

- Bilateral Total Hip Arthroplasty,

- Medical illness or known case of malignancy predisposed by radiation.

Post-operative radiological analysis of the version of acetabulum through X-ray images and CT images was performed. Pre \& post-operative scoring according to Modified Harris Hip Score (HHS), Western Ontario and McMaster Universities Osteoarthritis Index (WOMAC) and Oxford Hip Score (OHS) was documented.

Radiographic Assessment: Radiographs were taken with a tube-to-film distance of $120 \mathrm{~cm}$ by a computed digital radiographic system (Philips Bucky Primary Diagnost CS System 1000mA, November 2016 Hamburg, Germany).

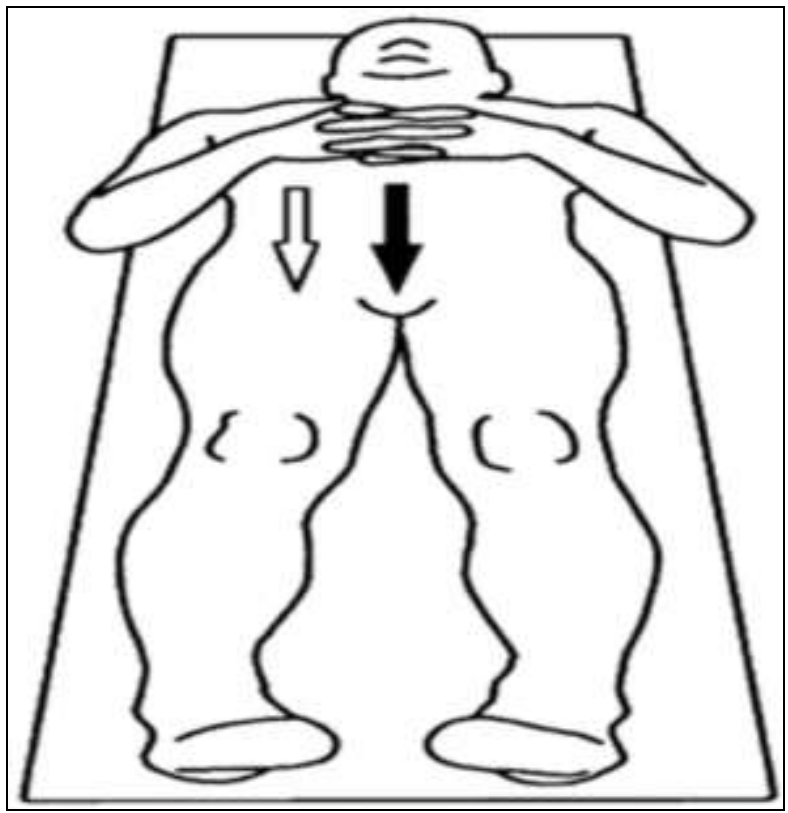

Fig 1: Technique of taking AP Radiograph

The patient was placed in the supine position with the bilateral hip joint in a neutral position. The radiation beam was centered on the pubic symphysis (black arrow) as shown in Figure 1 for an AP radiograph of the pelvis and was centered on the femoral head (white arrow) as shown in Figure 1 for the AP radiograph of the operated hip joint.

All CT scans included scanning by Antero-posterior tomogram as well as axial images of both acetabulum and femur. All angles were measured at CT work station (64 slice MDCT GE [General Electricals] Light Speed VCT XTE, April 2010, Wisconsin USA). The tomogram through the center of the acetabulum was selected for measurement of the acetabular anteversion angle, which is defined as the ventral orientation of the acetabulum related to the sagittal plane. A measuring point was assigned at the anterior edge of the acetabulum and a second at the posterior edge. The line connecting these points were drawn, and the angle formed by this line and the plane sagittal to the pelvis determined as the acetabular anteversion angle.

\section{Methods to calculate the version \\ Lewinnek's method}

Version $=\operatorname{arc} \sin (\mathrm{D} 1 / \mathrm{D} 2)$

D1 is the distance of the short axis of an ellipse drawn perpendicular to the long axis of the acetabular component; 
D2 is the distance of the long axis, which is considered the maximal diameter of the implant. Therefore, Lewinnek's method is more convenient for clinical practice and was used in the current study to calculate version angles.

The imaging data of CT scans for each case was anonymized prior to interpretation and stored in an online database where patient identification was removed, and the interpreting radiologists were blinded to prior measurements. The protocol was approved by our institution's ethics committee and written informed consent was obtained from each patient.

Statiscal Analysis: Patient characteristics were summarized using means and standard deviations for continuous variables and frequencies and percentages for discrete variables. Pearson's coefficients were determined to quantify the correlations between radiographic assessments of acetabular version on AP. The comparison of normally distributed continuous variables between the groups was performed using Student's t-test. Nominal categorical data between the groups were compared using the Chi-square test or Fisher's exact test whichever was found appropriate. Non-normal distribution continuous variables were compared using Mann Whitney $\mathrm{U}$ test, Odds ratio and $95 \%$ confidence interval was also calculated. For all statistical tests, a p-value less than 0.05 was taken to indicate a significant difference.

\section{Results}

In this study, anteversion angles calculated on AP Radiographs was $23.48^{\circ}$ (Range $\left.11-39^{\circ}\right)$, and as calculated on CT Scan had mean angle of $28.64^{\circ}$ (Range $\left.11.10-50.10^{\circ}\right)$. At final post-op follow-up $63.63 \%$ patients had excellent HHS, $23.63 \%$ patients had good whereas $610.90 \%$ patients had fair HHS post-operatively. Improved post-operative WOMAC score in $98.1 \%$ patients to excellent score. $78.1 \%$ patients had excellent OHS (40-48) post-operatively whereas $20 \%$ patients had good OHS (30-39). This was found to be statistically significant ( $\mathrm{p}$ value 0.038). The correlation between anteversion angles measured on CT scan and anteroposterior radiographs using Lewinnek's method indicated significant results ( $p$ value 0.046). Patients with higher BMI in our study had trend towards decreased anteversion angles. However, there was no significant correlation between BMI of the patient and anteversion angle. In this study, $40 \%$ patients had excellent Harris Hip score with anteversion angle in range of $24.1-36^{0}$ as measured on CT scan whereas $16.3 \%$ patients had excellent outcomes in range of $12.1-24^{0}$ of anteversion; $58.18 \%$ patients reported excellent WOMAC Score in anteversion angle in range of $24.1-36^{\circ}$, whereas $27.2 \%$ patients reported excellent outcomes in range between 12.1$24^{0}$ of anteversion; $50.9 \%$ patients reported excellent Oxford Hip Score with anteversion angle in range of $24.1-36^{\circ}$, whereas $16.3 \%$ of the patients reported excellent outcomes in range between $12.1-24^{0}$. In this study, only $30.90 \%$ the patients had anteversion in Lewinnek's safe zone i.e. $5-25^{\circ}$ on operated side whereas $70 \%$ patients had anteversion outside the Lewinnek's safe zone. On normal side $90.9 \%$ patients had anteversion in Lewinnek's safe zone. However, this correlation was non-significant. In present study, mean anteversion of native non-operated hip was $18.52^{\circ}$ (Range $6.3-35.2^{0}$ ) in male population whereas in females mean anteversion angle was $22.43^{\circ}$ (Range $11.4-29.5^{\circ}$ ). In current study group, $58.18 \%$ patients were males and mean angle of anteversion post-operative as measured on CT scan was $28.36^{0}$ (Range 16.4-50.1 ${ }^{\circ}$ ). $28.1 \%$ patients were in Lewinnek's safe zone. Whereas $41.8 \%$ patients were females with mean anteversion of $28.59^{\circ}$ (Range 11.1- 43.80); $34.78 \%$ patients had anteversion in Lewinnek's safe zone. The correlation between anteversion angles measured on CT scan and anteroposterior radiographs using Lewinnek's method indicated significant results ( $\mathrm{p}$ value 0.046 ). In our study there was no significant correlation between anteversion angles calculated on CT scan, X-rays and complications. We had one patient with femoral stem loosening and DVT was seen in the other patient; however, there was no significant correlation (p-value $0.302 \&$ p-value 0.140) respectively. Precision analysis was performed using intraclass correlation coefficients (ICCs) and spearman's rho. There was excellent intra-observer reliability for Lewinnek's method on anteroposterior radiographs $(\mathrm{ICC}=0.604, p<0.0001)$. On CT scan measurement spearman's rho coefficient was poor $(0.00$ to 0.20$)$, fair $(0.21$ to 0.40$)$, moderate $(0.41$ to 0.60$)$, good (0.61 to 0.80$)$ or excellent (0.81 to 1.00$)$. CT had a high intraand interobserver reliability, supporting this as the ideal method for measuring acetabular anteversion.

\section{Discussion}

Measuring the acetabular component position after total hip arthroplasty (THA) is commonly performed as part of the routine postoperative follow-up and as part of the assessment of the malfunctioning THA. The assessment of the acetabular component position is based on a combination of the inclination and anteversion angles. Pelvic computed tomography (CT) scans have been shown to be the most accurate assessment of the component position ${ }^{[11]}$. However, there still exists a dearth of literature regarding the effects of acetabular component positioning on hip functional outcome scores and till date patient satisfaction as a primary outcome in relation to acetabular version has not been done. In our study of 55 patients, the age ranged from 17 to 75 years of age with maximum number $49.09 \%$ of patients falling within 4964 years of age group. The mean age was 49.6 years. The mean age as reported by Sculco et al. ${ }^{[12]}$ and Park et al. ${ }^{[13]}$ in a study of 70 and 71 cases respectively reported a mean age of 58 years (Range 28-70 years) and 59.4 years (Range 24-78 years) respectively. Out of a total of 55 patients, there were 32 males $(58.18 \%)$ in our study and 23 females $(48.12 \%)$. Ha et al. ${ }^{[14]}$ conducted their study on 46 patients out of which male is to female ratio was 1:1. However, Nomura et al. [15] reported 84 patients out of which 63 were female and 21 were male patients. Pankaj et al. ${ }^{[16]}$ in their study of 29 patients had 17 male and 12 female patients. Sculco et al. in their study of 70 had 38 female patients. Park et al. had 47 male patients in their study of 71 patients. Nho et al. ${ }^{[17]}$ in their study of 36 patients had 19 males compared to 17 female patients. Lu et al. ${ }^{[18]}$ in their study of 60 had 35 male \& 25 female patients. All cases were of primary Total Hip Arthroplasty and the posterolateral approach was used in all cases as we are quite familiar with it. Lu et al. used the posterolateral approach in their study. Goyal et al. [19] measured the outcome of their study using a modified Hardinge approach on 1010 patients, whereas Callanan et al. [20] used direct lateral, posterolateral and anterolateral approach. McLawhorn et al. [21] used the posterolateral approach in their study. Consistent with other studies we found that the posterolateral approach has been frequently used in the past and is moderately accurate in attaining accurate combined anteversion and inclination angle within a target range. The mean BMI in our study population was $23.48 \mathrm{~kg} / \mathrm{m} 2.13(23.63 \%)$ patients were in the overweight category as per WHO guidelines, $2(3.63 \%)$ patients were in 
the obesity category. In our study, the patients with higher BMI had decreased anteversion angles. Brodt et al. ${ }^{[22]}$ from their study postulated that cup anteversion significantly correlated with BMI; this corresponded with a reduction of anteversion by 3.4 degrees in the morbidly obese group compared with the normal-weight group and they concluded that the precision of cup positioning declines with increasing obesity. In our study, we used Lewinnek's method ${ }^{[23]}$ which was found to be consistent with CT scans values of measured anteversion angles. Nishino et al. ${ }^{[24]}$ postulated that AP radiographs are better than cross-table lateral radiographs. Nho et al. recommended the use of Lewwinek's, Woo and Morrey's method, Liaw's method for measuring the anteversion of acetabular component. Lu et al. used Lewwinek's method in their study and found that measurement of the orientation of acetabular components on plain AP radiographs is reliable and accurate compared with measurement on CT. Maheshwari et al. ${ }^{[25]}$ in their study on Indian population measured mean acetabular anteversion of normal hips to be $19^{0}$ (Range $8-35^{\circ}$ ) which was comparable to measured angle on normal non operated hips in our study. Murphy et al. ${ }^{[26]}$ The best functional outcomes as reported by the patients on the basis of functional scores in our study was in the wider range of $12.1-36^{\circ}$ of anteversion, which is similar to studies done in recent past. Therefore, our study supports the notion that Lewinnek's safe zone is not a reliable predictor of stability and there is no absolute specific safe zone for acetabular component positioning. We also found that in the freehand placement of acetabular cups only a minority of the cups could be placed in Lewinnek's safe zone as seen in 17 $(30.90 \%)$ of the patients. Demonstrated that the historic Lewinnek safe zone is not a reliable predictor of future stability.

Table 1: Table shows in ideal anteversion and study

\begin{tabular}{|c|c|}
\hline Study & Ideal Anteversion \\
\hline Lewwinek et al. & $15^{\circ} \pm 10^{\circ}$ \\
\hline Dorr et al. & $15^{\circ} \pm 15^{\circ}$ \\
\hline McCollum and Gray & $30^{\circ} \pm 10^{\circ}$ \\
\hline Biedermann et al. & $15^{\circ} \pm 10^{\circ}$ \\
\hline Barrack et al. & $20^{\circ} \pm 10^{\circ}$ \\
\hline Widmer and Zurfluh & $20^{\circ}-28^{\circ}$ \\
\hline Present study & $30 \pm 6^{\circ}$ \\
\hline
\end{tabular}

In this study, $35(63.63 \%)$ had excellent and $13(23.63 \%)$ patients good whereas $6(10.90 \%)$ patients had fair HHS at final follow-up. Anteversion angle in range of 24.1-360 as measured on CT was associated with excellent outcomes. Even in acetabular anteversion range of $36.1-600,3(5.45 \%)$ patients had excellent HHS, whereas only $11(20 \%)$ patients lying in Lewinnek's safe zone had excellent functional outcomes. Goyal et al. demonstrated significant improvement in HHS total score. The absolute position of the cup showed a very weak yet significant positive correlation in between cup anteversion and HHS pain $(\mathrm{p}=0.01)$, HHS function $(\mathrm{p}=0.001)$ and HHS total score $(\mathrm{p}=0.001)$. The mean anteversion was $21.8 \pm 11.80$ in their study compared to mean of $28.64^{0}$ (Range $11.10-50.10^{\circ}$ ) in our study. The effect of the absolute and relative position of the acetabular component in total hip replacements on functional outcomes has not been studied in the literature. The ideal position of the acetabular component is still debated in the literature. Previous studies have not examined patient satisfaction as a primary outcome. Our study shows significant improvement in post-operative HHS in terms range of motion, pain relief as reported by patients.
Our study also shows that the Lewinnek's "safe zone" does not have any direct effect on patient outcome score. Majority of patients in anteversion angles range of 12.1-360 in our study had good to excellent outcomes which are comparable to a study done by Goyal et al. 91 Within the range of different acetabular cup positions studied, patient satisfaction was not significantly affected. Nevertheless, precision in cup position remains important for tribology and hip stability. Goyal et al. reported WOMAC Scores in their study; WOMAC pain ( $\mathrm{p}=0.02)$, WOMAC physical function $(\mathrm{p}=0.01)$ and WOMAC total $(\mathrm{p}=0.02)$ which was significant. The mean anteversion was $21.8 \pm 11.80$ in their study. According to them, disease-specific instruments such as WOMAC and HHS allow a focused and comprehensive assessment of treatment effects. Quintana et al. ${ }^{[27]}$ have shown that the minimal clinically important difference (MCID) for WOMAC is between 25.91 and 29.26 points for patients with primary total hip arthroplasty. In our study, anteversion angles in the range of $24.1-36^{0}$ which is well outside the Lewinnek's safe zone reported excellent WOMAC scores which is comparable to a study done by Goyal et al. In the present study, 43(78.1\%) patients had excellent OHS post-operatively compared to their pre-operative OHS which was statistically significant (p-value 0.038). Sculco et al. reported better OHS outcomes in the range of anteversion angles of $25 \pm 5^{\circ}$. In our study, anteversion angles in range $30 \pm 6^{\circ}$ had excellent outcomes which are fairly comparable to the study done by Sculco et al. and Grammatopoulos et al. Oxford hip score has not been widely used previously to assess functional outcome in relation to acetabular anteversion angles, as it is a patient based tool, its use may prove valuable to assess functional outcome. In our study, the mean anteversion angle as calculated by Lewinnek's method on AP radiographs was $23.48^{\circ}$ compared to mean angle of $28.64^{\circ}$ on CT scan, the difference was statistically significant ( $p$-value 0.046 ). In our study, possible reason for difference in measurements of anteversion angles could possibly be due to pelvic tilt (PT) although every possible precaution was taken to eliminate it. It has been found that there is a linear relationship between changes in PT and functional anteversion, anterior PT reduces functional component anteversion by approximately $0.74^{\circ}$ per degree of PT, while posterior PT increases it by the same amount.82 This is supported by study done by Tannast $e t$ al. [28] who analysed the influence of tilting, rotation, and obliqueness of the pelvis on measurements obtained from routine AP radiographs and Maratt et al. [29]. Moreover, measurement done on radiographs in our study was manual using a goniometer which also could be a possible reason to contribute error in measurement when compared to more precise measurement done in CT console. Moreover, different methods for measuring anteversion are based on those different definitions of cup orientation. On the transectional plane on CT, anteversion is anatomic, but when calculated from plain AP radiographs using the method of Lewinnek et al. it is radiographic.

In our study, anteversion angles ranged from $11.10-50.10^{0}$ with $17(30.90 \%)$ patients in Lewinnek safe zone. Saxler et al. ${ }^{[30]}$ concluded that safe position as defined by Lewinnek et al. was only achieved in a minority of the cups that were implanted freehand. Danoff et al. ${ }^{\text {[31] }}$ advocated a sweet spot safe zone of 17.10 of anteversion. Abdel et al. ${ }^{[32]}$ concluded that the historical target values for cup inclination and anteversion may be useful but should not be considered a safe zone given that the majority of these contemporary THAs that dislocated were within those target values. Goyal et al. 
concluded that "safe zone" has no effect on patient-reported functional outcomes which are similar to our study as the majority of patients with excellent functional outcomes were within the range of $24.1-36^{\circ}$ of anteversion which is outside the historic Lewinnek's safe zone. Similarly, patients outside Lewinnek's safe zone too had excellent and good HHS, WOMAC and OHS. We too believe that ideal cup position varies from patient to patient. More studies are needed to further redefine the concept of safe zone or the range of safe zone needs to be more comprehensive or elaborate. Our study is the first to calculate acetabular anteversion in patients operated by posterolateral approach with freehand technique. Also we have assessed functional outcomes by HHS, WOMAC and OHS and correlated this with version angles calculated on AP radiographs using Lewinnek's method and CT scan. There are limitations of this study. The first limitation was that we used goniometer to measure angles on radiographs instead of more precise computer software. We used free hand techniques on the basis of visual cues instead navigation controlled implantation of acetabular cup or mechanical alignment guides. There is a bias in the measurements of anteversion using radiographs which is inevitable when measuring a $3 \mathrm{D}$ object with a $2 \mathrm{D}$ projection. In our study, AP radiographs have also been used for measurement of acetabular component anteversion. However, this use of AP radiographs also has some disadvantages. It is difficult to identify the apex of the ellipse on AP radiographs, depending on the articulation type or the extent of the anteversion. If we had used the template software, we might have been able to more accurately measure anteversion on AP radiographs. In present study, we did not study the impact of pelvic mobility. There might be a difference in the values measured with a hypermobile versus a stiff pelvis. We also recognize that our conclusions regarding the effect of patient characteristics, specifically sex and BMI, are not definitive because they may indicate that our study was underpowered to establish such relationships. The patient's position during radiography influences the measurements. We largely eliminated the patient positioning variables (pelvic tilt and rotation) which produce error in clinical practice. These limitations must be taken into consideration when our results are applied in clinical practice. There is no gold standard for validation of radiographic or CT-based values in vivo. Because previous studies have shown that acetabular component orientation can be measured accurately by $\mathrm{CT}$ methods, we used 3-D CT measurements as the reference standard. Our sample size was small. The study design was reviewed by our institutional review board, which restricted the case number because of the radiation hazard and cost of CT scans, further we did not perform multiple CT scans on the same patients because of concern for radiation exposure; thus, we have no repeatability data for the CT scans. Our study is one of the few studies done in the past which measures patient reported functional outcomes as a function of anteversion angle. We found that the historical target values for cup anteversion defined by Lewinnek et al. may be useful but should not be considered a safe zone in the sense that positioning the acetabular component within these parameters does not preclude complications. Defining the optimal cup position is challenging. A good understanding of anatomic, patient and implant related factors that affect the "optimal" cup position is mandatory. Creating a stable THA remains a balancing act among appropriate component positioning taking into account individual patient bony and muscular anatomy in both the static and dynamic state, soft tissue balance and tensioning, and appropriate aftercare and rehabilitative efforts. Due to the variety of study designs, surgical approaches, and patient populations identified, it is difficult to draw broad conclusions regarding a definitive target zone for cup positioning in THA. The target zone for cup placement is probably influenced by several other factors, so the ideal target zone for each patient may vary depending on these factors. Considering the advantages of plain radiography, including low cost, low radiation level, and convenience for clinical follow-up and assessment of prosthesis position we recommend the use of Lewinnek's method as they provide reproducible and accurate data compared with CT. We believe that the safe zone should be tailored to the surgical approach used. One must remember that hip joint stability is multifactorial and dependent on additional variables such as femoral stem position, abductor complex quality and proper tensioning, and careful hip capsule closure. A properly positioned acetabular cup in isolation will not guarantee success. Intentionally we inserted the acetabular cup in more anteversion. It has been observed that $20-25 \%$ of patients subject their hip to deep seating and even go for squatting against advice. To avoid dislocation, the anteversion was kept about 100 above the normal. If we had to err, it was towards more anteversion. This was coupled with myocapsuloplasty, as a result we had no dislocation in our patients. Further research is encouraged to investigate the ability of emerging technologies to assist surgeons in optimally positioning the acetabular components. Functional outcome measurements with improved resolution may be of great importance for clinical research in the future.

\section{Conclusion}

In present study patients operated by posterolateral approach with freehand technique of acetabular cup implantation. Majority 39(69.09\%) of patients had excellent functional outcomes in a range of 11.1 - 360 of anteversion compared to Lewinnek's safe zone. It suggests that there is flexibility in positioning the acetabular component than previously believed. If one has to err, it should be towards more anteversion. Infact to avoid dislocation, more anteversion is required to guard against unwarranted activities on part of the patient. The anteroposterior radiographs can be used as a surrogate method for measuring the anteversion angle compared to $\mathrm{CT}$ scan as it avoids radiation exposure and lessens the cost.

\section{References}

1. Bhaskar D, Rajpura A, Board T. Current concepts in acetabular positioning in total hip arthroplasty. Indian $\mathbf{J}$ Orthop. 2017;51:386-96.

2. Bosker BH, Verheyen CC, Horstmann WG, Tulp NJ. Poor accuracy of freehand cup positioning during total hip arthroplasty. Arch Orthop Trauma Surg. 2007;127(5):375-379.

3. Lewinnek GE, Lewis JL, Tarr R, Compere CL, Zimmerman JR. Dislocations after total hip-replacement arthroplasties. J Bone Joint Surg Am. 1978;60:217-20.

4. Schmalzried TP, Shepherd EF, Dorey FJ, Jackson WO, dela Rosa M, Fa'vae F, et al. The John Charnley Award. Wear is a function of use, not time. Clin Orthop Relat Res. 2000;381:36-46.

5. Hedlundh U, Ahnfelt L, Hybbinette $\mathrm{CH}$, Wallinder L, Weckstro"m J, Fredin H. Dislocations and the femoral head size in primary total hip arthroplasty. Clin Orthop Relat Res. 1996;333:226-233. 
6. Maruyama M, Feinberg JR, Capello WN, D’Antonio JA. The Frank Stinchfield Award: Morphologic features of the acetabulum and femur: anteversion angle and implant positioning. Clin Orthop Relat Res. 2001;393:52-65.

7. Murray DW. The definition and measurement of acetabular orientation. J Bone Joint Surg Br. 1993;75:228-32.

8. Woo RYG, Morrey BF. Dislocations after Total Hip Arthroplasty. J Bone Joint Surg Am. 1982;64A:12951306.

9. McCollum DE, Gray WJ. Dislocation after total hip arthroplasty: Causes and prevention. Clin Orthop Relat Res. 1990;261:159-70.

10. Harris WH. Edge loading has a paradoxical effect on wear in metal-on-polyethylene total hip arthroplasties. Clin Orthop Relat Res. 2012;470:3077-3082.

11. Kalteis T, Handel M, Herold T, Perlick L, Paetzel C, Grifka J. Position of the acetabular cup accuracy of radiographic calculation compared to CT-based measurement. European Journal of Radiology. 2006;58(2):294-300.

12. Sculco PK, McLawhorn AS, Carroll KM, McArthur BA, Mayman DJ. Anteroposterior Radiographs Are More Accurate than Cross-Table Lateral Radiographs for Acetabular Anteversion Assessment: A Retrospective Cohort Study. HSS Journal. 2016;12(1):32-8.

13. Park YS, Shin WC, Lee SM, Kwak SH, Bae JY, Suh KT. The best method for evaluating anteversion of the acetabular component after total hip arthroplasty on plain radiographs. J Orthop Surg Res. 2018;13(1):66.

14. Ha YC, Yoo JJ, Lee YK et al. Acetabular component positioning using anatomic landmarks of the acetabulum. Clin Orthop Relat Res. 2012;470(12):3515-23.

15. Nomura $T$, Naito $M$, Nakamura $Y$, Ida T, Kuroda D, Kobayashi T, et al. An analysis of the best method for evaluating anteversion of the acetabular component after total hip replacement on plain radiographs. Bone Joint J. 2014;96-B(5):597-603.

16. Pankaj A, Mittal A, Chawla A. The validity and reproducibility of cross table radiographs compared with CT scans for the measurement of anteversion of the acetabular component after total hip arthroplasty. Bone Joint J. 2017;99-B(8):1006-11.

17. Nho JH, Lee YK, Kim HJ, Ha YC, Suh YS, Koo KH. Reliability and validity of measuring version of the acetabular component. J Bone Joint Surg Br. 2012;94$\mathrm{B}(1): 32-6$.

18. Lu M, Zhou YX, Du H, Zhang J, Liu J. Reliability and Validity of Measuring Acetabular Component Orientation by Plain Anteroposterior Radiographs. Clin Orthop Relat Res. 2013;471(9):2987-94.

19. Goyal P, Lau A, Naudie DD, Teeter MG, Lanting BA, Howard JL. Effect of Acetabular Component Positioning on Functional Outcomes in Primary Total Hip Arthroplasty. J Arthroplasty. 2017;32(3):843-8.

20. Callanan MC, Jarrett B, Bragdon CR, Zurakowski D, Rubash HE, Freiberg AA, et al. The John Charnley Award: Risk Factors for Cup Malpositioning: Quality Improvement through a Joint Registry at a Tertiary Hospital. Clin Orthop Relat Res. 2011;469(2):319-29.

21. McLawhorn AS, Sculco PK, Mayman DJ. Targeting a New Safe Zone: A Step in the Development of PatientSpecific Component Positioning for Total Hip Arthroplasty. American Journal of Orthopedics. 2015;6:270-76.
22. Brodt S, Jacob B, Windisch C, Seeger J, Matziolis G. Morbidly Obese Patients Undergoing Reduced Cup Anteversion through a Direct Lateral Approach. J Bone Joint Surg Br. 2016;98(9):729-34.

23. Lewinnek GE, Lewis JL, Tarr R, Compere CL, Zimmerman JR. Dislocations after total hip-replacement arthroplasties. J Bone Joint Surg Am. 1978;60:217-20.

24. Nishino H, Nakamura S, Arai N, Matsushita T. Accuracy and Precision of Version Angle Measurements of the Acetabular Component after Total Hip Arthroplasty. J Arthroplasty. 2013;28(9):1644-7.

25. Maheswari A, Zlowodzki P, Siram M, Jain G, AK. Femoral neck Anteversion, cetabular Anteversion and combined anteversion in normal Indian adult population: A computed tomographic study. Indian J Orthop. 2010;44:277-282.

26. Murphy WS, Yun HH, Hayden B, Kowal JH, Murphy SB. The Safe Zone Range for Cup Anteversion Is Narrower Than for Inclination in THA. Clin Orthop Relat Res. 2018;476(2):325-35.

27. Quintana JM, Escobar A, Bilbao A, Arostegui I, Lafuente I, Vidaurreta I. Responsiveness and clinically important differences for the WOMAC and SF-36 after hip joint replacement. Osteoarthritis and Cartilage. 2005;13(12):1076-83.

28. Tannast M, Langlotz U, Siebenrock K-A, Wiese M, Bernsmann K, Langlotz F. Anatomic Referencing of Cup Orientation in Total Hip Arthroplasty. Clin Orthop Relat Res. 2005;436:144-50.

29. Maratt JD, Esposito CI, McLawhorn AS, Jerabek SA, Padgett DE, Mayman DJ. Pelvic Tilt in Patients Undergoing Total Hip Arthroplasty: When Does It Matter? J Arthroplasty. 2015;30(3):387-91.

30. Stem ES, O’Connor MI, Kransdorf MJ. Computed tomography analysis of acetabular anteversion and abduction. Skeletal Radiol. 2006;35:385-9.

31. Danoff JR, Bobman JT, Cunn G, Murtaugh T, Gorroochurn P, Geller JA, et al. Redefining the Acetabular Component Safe Zone for Posterior Approach Total Hip Arthroplasty. J Arthroplasty. 2016;31(2):50611.

32. Abdel MP, von Roth P, Jennings MT, Hanssen AD, Pagnano MW. What Safe Zone? The Vast Majority of Dislocated THAs Are Within the Lewinnek Safe Zone for Acetabular Component Position. Clin Orthop Relat Res. 2016;474(2):386-91. 